\title{
Erratum
}

\section{Determinants of Laplacians on Surfaces of Finite Volume}

Commun. Math. Phys. 119, 443-451 (1988)

\section{Isaac Efrat}

Department of Mathematics, University of Maryland, College Park, MD 20742, USA

Received January 10, 1991

The two formulae on p. 447 that describe the contribution of the continuous spectrum to the theta kernel are incorrect. We therefore need to redefine the kernel according to what is forced by the trace formula. We let

$$
\theta(t)=\sum_{\sigma \in S_{1}} e^{-\sigma(1-\sigma) t}+\frac{1}{2 \pi} \int_{-\infty}^{\infty}-\frac{\phi^{\prime}}{\phi}(1 / 2+i r) e^{-\left(1 / 4+r^{2}\right) t} d t,
$$

which can also be written as

$$
\sum_{\sigma \in S_{1}} e^{-\sigma(1-\sigma) t}+\sum_{e \in S_{2} \cup S_{3}} \frac{1-2 \beta}{2 \pi} \int_{-\infty}^{\infty} \frac{e^{-\left(1 / 4+r^{2}\right) t}}{(r-\gamma)^{2}+(1 / 2-\beta)^{2}} d r .
$$

Here for $\varrho \in S_{3}$ we let $\beta=\eta, \gamma=0$. The zeta function is then

$\zeta(\mathrm{s}, \mathrm{w})=\sum_{\sigma \in S_{1}}(\sigma(1-\sigma)-s(1-s))^{-w}+\frac{1}{2 \pi} \int_{-\infty}^{\infty}-\frac{\phi^{\prime}}{\phi}(1 / 2+i r)\left(\left(1 / 4+r^{2}\right)-s(1-s)\right)^{-w} d r$.

The propositions that follow are unchanged. If we define as before

$$
\operatorname{det}^{2}(\Delta-s(1-s))=e^{-\left.\frac{\partial}{\partial w} \zeta(w, s)\right|_{w=0}},
$$

then our main theorem is slightly simplified. It reads

$$
\operatorname{det}^{2}(\Delta-s(1-s))=Z(s)^{2} Z_{\infty}(s)^{2} \Gamma(s+1 / 2)^{-2 h_{1}}(2 s-1)^{A} e^{B(2 s-1)^{2}+C(2 s-1)+D} .
$$

We remark that the determinant squared which is studied in the paper (that now depends on the kernel satisfying the favorable asymptotics given in Proposition 1) is invariant with respect to $s \rightarrow 1-s$, while our new determinant, in spite of the notation, is not. On the other hand, the latter has the advantage that it is naturally given as a square.

We wish to acknowledge W. Müller for finding the error, as well as D. Hejhal and S. Wolpert for some useful discussions. 